Canad. Math. Bull.Vol. 35 (1), 1992 pp. 40-45

\title{
NOTE ON THE SPACE BMOA
}

\author{
JUN SOO CHOA
}

Abstract. Let $D$ be the unit disc in the complex plane. It is shown that

(i) for $0<p<2$, there exists an analytic function $f \in \mathrm{BMOA}$ for which

$$
\int_{D}\left|f^{\prime}(z)\right|^{p}\left(1-|z|^{2}\right)^{p-2}\left(1-\left|\varphi_{a}(z)\right|^{2}\right) d x d y=\infty \text { when }|a|<1,
$$

and

(ii) for $2<p<\infty$, there exists an analytic function $f \notin$ BMOA for which

$$
\sup _{a \in D} \int_{D} \mid f^{\prime}(z)^{p}\left(1-|z|^{2}\right)^{p-2}\left(1-\left|\varphi_{a}(z)\right|^{2}\right) d x d y<\infty .
$$

This settles the question of Stroethoff [5] on BMOA.

1. Introduction. Let $D=\{z \in C:|z|<1\}$ be the unit disc in the complex plane. For $a \in D$, define a Möbius transformation $\varphi_{a}: D \rightarrow D$ by

$$
\varphi_{a}(z)=\frac{a-z}{1-\bar{a} z}, \quad z \in D
$$

The Bloch space $\mathcal{B}$ is the set of all analytic functions $f$ on $D$ for which

$$
\|f\|_{\mathcal{B}}=\sup _{a \in D}\left|\left(f \circ \varphi_{a}\right)^{\prime}(0)\right|<\infty
$$

Contained in the Bloch space is the little Bloch space $\mathcal{B}_{0}$, which is by definition the set of all analytic functions $f$ on $D$ for which $\left|\left(f \circ \varphi_{a}\right)^{\prime}(0)\right| \rightarrow 0$ as $|a| \rightarrow 1-$. Recently, Stroethoff [5] obtained the following Möbius-invariant characterization for the (little) Bloch space as a part of his results.

THEOREM A. Let $0<p<\infty$ and let $f$ be an analytic function on $D$. Then

$$
f \in \mathcal{B} \Leftrightarrow \sup _{a \in D} \int_{D}\left|f^{\prime}(z)\right|^{p}\left(1-|z|^{2}\right)^{p-2}\left(1-\left|\varphi_{a}(z)\right|^{2}\right)^{2} d x d y<\infty ;
$$

and

$$
f \in \mathcal{B}_{0} \Leftrightarrow \int_{D}\left|f^{\prime}(z)\right|^{p}\left(1-|z|^{2}\right)^{p-2}\left(1-\left|\varphi_{a}(z)\right|^{2}\right)^{2} d x d y \rightarrow 0 \text { as }|a| \rightarrow 1-.
$$

This research was partially supported by TGRC-KOSEF

Received by the editors January 8, 1991; revised April 30, 1991.

AMS subject classification: Primary 30D55.

Key words and phrases: Bloch space, bounded mean oscillation

(c) Canadian Mathematical Society 1992. 
The space BMOA ("Bounded Mean Oscillation", see [1]) is the set of all analytic functions $f$ on $D$ for which

$$
\|f\|_{\mathrm{BMOA}}=\sup _{a \in D}\left\|f \circ \varphi_{a}-f(a)\right\|_{H^{2}}<\infty .
$$

Contained in BMOA is the subspace VMOA ("Vanishing Mean Oscillation"), the set of all analytic functions $f$ on $D$ for which $\left\|f \circ \varphi_{a}-f(a)\right\|_{H^{2}} \rightarrow 0$ as $|a| \rightarrow 1-$. As is well known (see [2], for example), the space BMOA (resp., VMOA) has the following Möbius-invariant characterization: If $f$ is an analytic function on $D$, then

$$
f \in \mathrm{BMOA} \Leftrightarrow \sup _{a \in D} \int_{D}\left|f^{\prime}(z)\right|^{2}\left(1-\left|\varphi_{a}(z)\right|^{2} d x d y<\infty\right.
$$

and

$$
f \in \mathrm{VMOA} \Leftrightarrow \int_{D}\left|f^{\prime}(z)\right|^{2}\left(1-\left|\varphi_{a}(z)\right|^{2}\right) d x d y \rightarrow 0 \text { as }|a| \rightarrow 1-.
$$

The Bloch space and the space BMOA share many analogous properties, as do the little Bloch space and the space VMOA. Motivated by this fact and the observation of the equivalences (A.1) for the Bloch space (resp., (A.2) for the little Bloch space) when $p=2$ and (i) for BMOA (resp., (ii) for VMOA), Stroethoff [5] asked the following:

QUESTIONS. Let $0<p<\infty$ and let $f$ be an analytic function on $D$. Are the following true?

$$
f \in \mathrm{BMOA} \Leftrightarrow \sup _{a \in D} \int_{D}\left|f^{\prime}(z)\right|^{p}\left(1-|z|^{2}\right)^{p-2}\left(1-\left|\varphi_{a}(z)\right|^{2}\right) d x d y<\infty
$$

and

(Q. 2) $f \in \mathrm{VMOA} \Leftrightarrow \int_{D}\left|f^{\prime}(z)\right|^{p}\left(1-|z|^{2}\right)^{p-2}\left(1-\left|\varphi_{a}(z)\right|^{2}\right) d x d y \rightarrow 0$ as $|a| \rightarrow 1-$. In this paper, we settle these questions in the negative.

2. Known results. We collect some known facts which will be used in the proof of our main theorem.

The following proposition gives a way of getting BMOA-functions by using a lacunary series.

PROPOSITION 1 [3, PP. 44-45]. Let $n_{1}<n_{2}<\cdots$ be a sequence of positive integers satisfying $\inf _{k} n_{k+1} / n_{k}>1$ for all $k$ and let $f(z)=\sum_{k=1}^{\infty} a_{k} z^{n_{k}}$. Then the following statements are equivalent:

(a) $f \in H^{2}$ i.e. $\sum_{k=1}^{\infty}\left|a_{k}\right|^{2}<\infty$;

(b) $f \in$ BMOA;

(c) $f \in$ VMOA.

The next lemma is taken from [4, p. 339].

LEMMA 2. Let $s_{k}$ be a sequence of positive numbers and $s_{k} / s_{k+1} \rightarrow 0$ as $k \rightarrow \infty$. Then

$$
\sum_{j=1}^{k-1} s_{j}=o\left(s_{k}\right) \text { as } k \rightarrow \infty .
$$


3. Main theorem. In what follows, to save some writing, we use the notation

$$
I_{p}(f ; a)=\int_{D}\left|f^{\prime}(z)\right|^{p}\left(1-|z|^{2}\right)^{p-2}\left(1-\left|\varphi_{a}(z)\right|^{2}\right) d x d y
$$

for an analytic function $f$ on $D$ and $a \in D$.

The following is our main theorem.

THEOREM 3. (i) If $0<p<2$, then there exists an analytic function $f \in \mathrm{BMOA}$ for which

$$
I_{p}(f ; a)=\infty \text { when }|a|<1
$$

(ii) If $2<p<\infty$, then there exists an analytic function $f \notin \mathrm{BMOA}$ for which

$$
\sup _{a \in D} I_{p}(f ; a)<\infty
$$

REMARK. It can be proved by elementary calculations (see [5], for example) that the condition $\sup _{a \in D} I_{p}(f ; a)<\infty$ is sufficient for the containment $f \in$ BMOA when $0<$ $p<2$ and the condition $\sup _{a \in D} I_{p}(f ; a)<\infty$ is necessary in order to have $f \in$ BMOA when $2<p<\infty$.

ProOF. Fix $0<p<2$, and let $n_{k}=(k !)^{2}$ and define

$$
f(z)=\sum_{k=1}^{\infty} k^{-1 / p} z^{n_{k}}
$$

Let

$$
A_{k}=\left\{z \in D: 1-2 / n_{k} \leq|z| \leq 1-1 / n_{k}\right\} \quad(k=2,3, \ldots)
$$

be the annulus in $D$. If $z \in A_{k}$, then

$$
\begin{aligned}
\left|f^{\prime}(z)\right| \geq\left|z f^{\prime}(z)\right| & =\left|\sum_{j=1}^{\infty} j^{-1 / p} n_{j} z^{n_{j}}\right| \\
& \geq k^{-1 / p} n_{k}|z|^{n_{k}}-\sum_{j=1}^{k-1} j^{-1 / p} n_{j}-\sum_{j=k+1}^{\infty} j^{-1 / p} n_{j}|z|^{n_{j}} \\
& =(\mathrm{I})-(\mathrm{II})-(\mathrm{III}) .
\end{aligned}
$$

Since $\left(1-2 / n_{k}\right)^{n_{k}}$ increases with increasing $n_{k}$. It is zero, when $n_{k}=2$. However for $k \geq 3$ we have $n_{k} \geq 36$ and $\left(1-2 / n_{k}\right)^{n_{k}}>1 / 8$. It follows that

$$
\text { (I) } \geq k^{-1 / p} n_{k}\left(1-2 / n_{k}\right)^{n_{k}} \geq \frac{1}{8} k^{-1 / p} n_{k} .
$$

If we note that

$$
k^{-1 / p} n_{k} /(k+1)^{-1 / p} n_{k+1} \rightarrow 0 \text { as } k \rightarrow \infty
$$


we have by Lemma 2

$$
\text { (II) }=o\left(k^{-1 / p} n_{k}\right) \text {. }
$$

Suppose that $n$ is a positive integer and $0<x<1$. From the binomial theorem we deduce

$$
1=(x+1-x)^{n+2} \geq \frac{1}{2}(n+1)(n+2) x^{2}(1-x)^{n}>\frac{1}{2}(n x)^{2}(1-x)^{n} .
$$

So that

$$
(1-x)^{n}<2 /(n x)^{2}
$$

Applying this inequality we deduce that

$$
\begin{aligned}
\sum_{j=k+1}^{\infty} j^{-1 / p} n_{j}\left(1-1 / n_{k}\right)^{n_{j}} & \leq 2 k^{-1 / p} n_{k}^{2} \sum_{j=k+1}^{\infty} 1 / n_{j} \\
& \leq 2 k^{-1 / p} n_{k} \sum_{j=k+1}^{\infty} n_{j-1} / n_{j} \\
& \leq 2 k^{-1 / p} n_{k} \sum_{j=k+1}^{\infty} j^{-2} .
\end{aligned}
$$

Since the series $\sum_{j=1}^{\infty} j^{-2}$ converges, it follows that

$$
\text { (III) }=o\left(k^{-1 / p_{n}}\right)
$$

Thus if $k_{0}$ is large enough, then for $k \geq k_{0}$

$$
\left|f^{\prime}(z)\right|>n_{k} /\left(10 k^{1 / p}\right), \quad z \in A_{k} .
$$

Note that the area of $A_{k}$ is

$$
\pi\left[\left(1-1 / n_{k}\right)^{2}-\left(1-2 / n_{k}\right)^{2}\right]>\pi / n_{k},
$$

and $1-|z|^{2} \geq 1-|z|>2 / n_{k}$ for $z \in A_{k}$. Then we see that

$$
\begin{aligned}
\frac{1+|a|}{1-|a|} \int_{D}\left|f^{\prime}(z)\right|^{p}\left(1-|z|^{2}\right)^{p-2}\left(1-\left|\varphi_{a}(z)\right|^{2}\right) d x d y \\
\quad \geq \int_{D}\left|f^{\prime}(z)\right|^{p}\left(1-|z|^{2}\right)^{p-1} d x d y \\
\quad \geq \sum_{k_{0}}^{\infty} \frac{n_{k}^{p}}{10^{p} k}\left(\frac{2}{n_{k}}\right)^{p-1} \frac{\pi}{n_{k}} \\
\quad=\frac{\pi}{2 \cdot 5^{p}} \sum_{k_{0}}^{\infty} \frac{1}{k}=\infty .
\end{aligned}
$$

Therefore (3.1) is satisfied. However since $\sum\left(k^{-1 / p}\right)^{2}<\infty$ for $0<p<2$, we have $f \in$ BMOA by Proposition 1. This shows (i). 
If $2<p<\infty$ we define $f(z)=\sum_{k=1}^{\infty} k^{-1 / 2} z^{2^{k}}$. Then

$$
\left|r f^{\prime}\left(r e^{i \theta}\right)\right| \leq \sum_{k=1}^{\infty} 2^{k} k^{-1 / 2} r^{2^{k}}
$$

If we observe that

$$
2^{k} r^{2^{k}} \leq 2 \sum_{2^{k-1}<l \leq 2^{k}} r^{l}
$$

then we have

$$
\left|r f^{\prime}\left(r e^{i \theta}\right)\right| \leq 2 \sum(\log l)^{-1 / 2} r^{l}
$$

It is not hard to see (for example, by comparing with an integral) that this last sum is bounded by a constant times

$$
1 /(1-r)(\log [1 /(1-r)])^{1 / 2} .
$$

We denote by $C$ an absolute constant, not necessarily the same on each occasion. It follows

$$
\begin{aligned}
& \int_{D}\left|f^{\prime}(z)\right|^{p}\left(1-|z|^{2}\right)^{p-2}\left(1-\left|\varphi_{a}(z)\right|^{2}\right) d x d y \\
&=\left(1-|a|^{2}\right)\left(\int_{|z| \leq 1 / 2}+\int_{|z| \geq 1 / 2}\right)\left|f^{\prime}(z)\right|^{p}\left(1-|z|^{2}\right)^{p-1} \frac{d x d y}{|1-\bar{a} z|^{2}} \\
&=\left(1-|a|^{2}\right) \max _{|z| \leq 1 / 2}\left(\left|f^{\prime}(z)\right|^{p}\left(1-|z|^{2}\right)^{p-1}\right) \int_{|z| \leq 1 / 2} \frac{d x d y}{|1-\bar{a} z|^{2}} \\
&+(1-|a|)^{2} \int_{1 / 2}^{1}\left|f^{\prime}\left(r e^{i \theta}\right)\right|^{p}\left(1-r^{2}\right)^{p-1} \int_{0}^{2 \pi} \frac{d \theta}{\left|1-\bar{a} r e^{i \theta}\right|^{2}} r d r \\
& \leq C\left(1-|a|^{2}\right)+C \frac{1-|a|^{2}}{1-|a|^{2} / 4} \int_{1 / 2}^{1} \frac{1}{(1-r)(\log [1 /(1-r)])^{p / 2}} d r .
\end{aligned}
$$

The last integral converges, since $p>2$. Therefore (3.2) is satisfied. However, since $\sum\left(k^{-1 / 2}\right)^{2}=\infty$, it follows from Proposition 1 that $f \notin$ BMOA. This shows (ii). The proof is complete.

Observing the equivalence (a) and (c) of Proposition 1 and a careful look at the proof of Theorem 3 give the following:

THEOREM 4. (i) If $0<p<2$, then there exists an analytic function $f \in \mathrm{VMOA}$ for which

$$
\lim _{|a| \rightarrow 1-} I_{p}(f ; a) \neq 0
$$

(ii) If $2<p<\infty$, then there exists an analytic function $f \notin \mathrm{VMOA}$ for which

$$
\lim _{|a| \rightarrow 1-} I_{p}(f ; a)=0 .
$$

ACKNOWLEDGEMENT. The author wishes to express his sincere gratitude to the referee for the valuable comments and to Professor Hong Oh Kim for the helpful discussions. 


\section{REFERENCES}

1. A. Baernstein, Analytic Functions of Bounded Mean Oscillation. In: Aspects of Contemporary Complex Analysis, Academic Press, New York, 3-36.

2. J. B. Garnett, Bounded Analytic Functions, Academic Press, New York, 1981.

3. D. J. Hallenbeck and K. Samotij, On radial variation of bounded analytic functions, Complex Variables, 15(1990), 43-52.

4. P. B. Kennedy, On the derivative of a function of bounded characteristic, Quart. J. Math. Oxford, 15(1964), 337-341.

5. K. Stroethoff, Besov-type characterization for the Bloch space, Bull. Austral. Math. Soc., 39(1989), 405420.

Department of Mathematics Education

Sung Kyun Kwan University

Jongro-gu, Seoul 110-745

Korea 